\title{
Radio-Optical Analysis of Extended Radio Sources in the First Look Survey Field
}

\author{
C. M. Paulo ${ }^{1}$, I. Prandoni ${ }^{2}$, R. Morganti ${ }^{3}$, and C. M. Cress ${ }^{1}$ \\ ${ }^{1}$ University of the Western Cape, Department of Physics, Bellvile, 7535, South Africa \\ ${ }^{2}$ INAF-Instituto di Radioastronomia, Via Gobetti 101, I-40129 Bologna, Italy \\ ${ }^{3}$ ASTRON, Postbus 2, 7990 AA, Dwingwloo, The Netherlands
}

We combine $610 \mathrm{MHz}$ GMRT data, $1.4 \mathrm{GHz}$ VLA data, and 1.4 GHz WSRT observations, encompassing a $\sim 4$ square degree field centered on the verification strip of the Spitzer First Look Survey field, to study radio sources down to fluxes of about $0.1 \mathrm{mJy}$. The spectral index (Figure 1) analysis shows that the majority of multi-component sources are steep-spectrum sources. Nevertheless the spread in the spectral distribution is wide, with a significant number of ultra-steep, flat or inverted sources, possibly indicating a wider range of accretion modes in fainter samples. By cross-correlating 107 multicomponent radio sources with the optical catalogues of Marleau et al. (2007) and Papovich et al. (2006), 23 objects were identified. The measured redshifts span the range $0<z<1.8$ and peak at $z \sim 0.2$. According to their radio power, six of the identified objects are in the range of FR II sources while 17 are in the range of FR I sources. Further optical follow-up will allow a more complete census of the sub-mJy population and more information on AGN feedback from such sources.



Figure 1. Spectral index distribution. The vertical dashed line indicates the average spectral index value of the sample.

\section{References}

Papovich, C., Cool, R., Eisenstein, D., Le Floc'h, E., Fan, X., Kennicutt, R. C., Jr., Smith, J. T. D., Rieke, G. H., \& Vestergaard, M. 2006, AJ, 132, 231

Marleau, F. R., Fadda, D., Appleton, P. N., Noriega-Crespo, A., Im, M., \& Clancy, D. 2007, ApJ, 663, 218 